\title{
Distinctive clinicopathological features and disease-specific survival of adenoid cystic carcinoma and adenoid basal carcinoma in the lower female genital tract
}

\author{
DEYIN XING $^{1}$ and JIAYUN LU ${ }^{2}$ \\ ${ }^{1}$ Department of Pathology, The Johns Hopkins University School of Medicine, Baltimore, MD 21231, USA;
${ }^{2}$ Department of Epidemiology, The Johns Hopkins Bloomberg School of Public Health, Baltimore, MD 21205, USA
}

Received March 29, 2018; Accepted October 9, 2018

DOI: 10.3892/or.2018.6927

\begin{abstract}
Adenoid cystic carcinomas (ACCs) and adenoid basal carcinomas (ABCs) in the lower female genital tract are very rare. Data on the clinicopathologic features and survival outcomes of ACCs and ABCs in the lower female genital tract are limited to case reports and small case series studies. The present study systemically analyzed 233 cases, including 84 cervical ACCs, 78 cervical ABCs and 71 vulvar ACCs, to identify clinicopathologic features and survival factors in a population-based Surveillance, Epidemiology and End Results (SEER) study. Whereas cervical ACCs and ABCs tend to occur in the elderly (median, 72 and 69 years, respectively), vulvar ACCs commonly occurred in patients a decade younger (median, 59 years). The majority of patients with cervical $\mathrm{ABC}$ had localized disease and almost all received surgery. In contrast, cervical and vulvar ACC patients tended to have higher stage disease, and a significant proportion of these patients received radiotherapy, with or without surgery. The 5-year cause-specific survival (CSS) rates for patients with cervical ACC were $69.3 \%$, vulvar ACC $87.7 \%$ and cervical $\mathrm{ABC} 96.6 \%$. The 5-year overall survival (OS) rate for patients with cervical ACC was $59.2 \%$, significantly worse than that of cervical $\mathrm{ABC}(88.3 \% ; \mathrm{P}=0.002)$ and vulvar $\mathrm{ACC}(81.2 \%$; $\mathrm{P}=0.01)$. Increased age and high stage were significantly associated with a worse prognosis in patients with cervical and vulvar ACCs by univariate and multivariate analysis $(\mathrm{P}<0.05)$. Tumor stage was the only significant factor associated with 5 -year overall survival in patients with cervical ABC $(\mathrm{P}<0.05)$. The present data demonstrated that the distinctive clinicopathologic features and survival outcomes differed significantly
\end{abstract}

Correspondence to: Dr Deyin Xing, Department of Pathology, The Johns Hopkins University School of Medicine, 401 N. Broadway, Weinberg 2242, Baltimore, MD 21231, USA

E-mail: dxing2@jhmi.edu

Key words: adenoid basal carcinoma, adenoid cystic carcinoma, prognosis, SEER, uterine cervix, vulva among ACCs and ABCs in the lower female genital tract, thus providing a rationale for location/pathologic type-based treatment modalities.

\section{Introduction}

Adenoid cystic carcinoma (ACC) is an uncommon malignant neoplasm consisting of epithelial and myoepithelial cells arranged in variable patterns, including tubular, cribriform, and solid architecture. This tumor is thought to progress slowly but remains on a relentless clinical course $(1,2)$. The clinicopathologic features and behaviors of ACC are quite distinct, illustrated by frequent perineural invasion, local recurrence and late distant metastasis (3). The 5-year overall survival (OS) rate for ACC patients ranges from 64-91\% (1-6). Factors that influence survival include age, tumor size, tumor site, clinical stage, lymph node involvement, status of surgical margins and distant metastasis $(1,2,6,7)$. While radiotherapy has been reported to improve survival in cases with microscopic residual disease/positive surgical margins (8), the value of chemotherapy in this tumor appears to be limited $(4,9)$.

Although it mainly affects the major and minor salivary glands, ACC may also occur in a number of other locations including the uterine cervix and vulva $(10,11)$. Lower female genital tract tumors with adenoid cystic differentiation are very rare, accounting for less than $1 \%$ of all lower female genital tract malignancies (10). These tumors are thought to originate from the major vestibular (Bartholin) glands of the vulva and minor secretory glands or reserve cells of the uterine cervix, displaying similar histopathological features to ACC of non-genital tract sites. It has been proposed that ACCs of the uterine cervix and vulva can be sub-classified into two distinct groups based on the presence or absence of high-risk human papillomavirus (HPV) (12). The oncogenic mechanisms that underlie the development of HPV-unrelated ACC are thought to be similar to those of ACCs in other sites. In fact, nuclear factor NFIB-associated gene rearrangement is a frequent genetic event in vulvar ACCs, conferring a driving force to transform the cells (13). Unlike vulvar ACCs, our previous results demonstrated that cervical carcinomas with mixed differentiation, including adenoid cystic carcinomatous 
component, are etiologically associated with high-risk HPV and can be identified by diffuse p16 expression (12).

Adenoid basal tumors (epitheliomas/carcinomas) of the uterine cervix are also rare lower female genital tract neoplasms that display a mixed configuration of basaloid, squamous, and glandular morphology $(11,14,15)$. Similar to cervical ACC, adenoid basal tumors are also derived from high-risk HPV-infected reserve cells, sharing many morphologic features with ACC $(14,16)$. In fact, the two tumors were previously regarded as a single entity $(15,17)$. Lacking destructive infiltrative growth, adenoid basal epithelioma is usually an incidental finding in patients treated for a high-grade squamous intraepithelial lesion and usually behaves in a benign fashion (16,17). The terms 'adenoid basal carcinoma (ABC)' and 'adenoid basal epithelioma' are considered synonymous in the 2014 WHO Classification of the tumors of the uterine cervix (10). The presence of any invasive carcinoma subtype with $\mathrm{ABC}$ needs to be reported as a 'mixed carcinoma'.

Due to the rarity of ACC and ABC in the lower female genital tract, the majority of published literature comprises case reports or small case series studies with limited sample sizes $(14,15,17)$. Currently, the clinicopathologic features, treatment modalities and clinical outcomes of these tumors remain largely unknown, thus hampering the establishment of standard treatment protocols to guide clinical management. The Surveillance, Epidemiology and End Results (SEER) program of the National Cancer Institute is a public source of epidemiologic information on the cancer incidence and survival data from population-based cancer registries covering $\sim 28 \%$ of the population of the United States (18). Using data from the SEER program, the clinicopathologic features and survival outcomes of $\mathrm{ACC}$ and $\mathrm{ABC}$ of the uterine cervix were investigated. These features were also compared with those of vulvar ACC.

\section{Materials and methods}

Representative histologic images. The images of uterine cervical ACCs and ABCs and vulvar ACCs were taken from consultation cases at the Johns Hopkins Hospital (Baltimore, MD, USA). Details of methods for immunohistochemical analysis of p16 expression have been previously reported (12). In brief, formalin-fixed, paraffin-embedded tissue sections were used. Immunoperoxidase labeling was done with anti-p16(INK4a) (cat. no. 705-4793; Ventana Medical Systems, Inc., Tucson, AZ, USA) at a dilution of 1:500. All images were captured at a magnification of $\times 200$.

Patient selection. The SEER November 18, 2016 submission (18) is a public-use database that includes updated cancer incidence and population data associated by age, sex, ethnicity, year of diagnosis, geographic areas and cause of death. Data on patients with ACC and $\mathrm{ABC}$ of the lower female genital tract were obtained. All patients with a diagnosis of primary $\mathrm{ACC}$ and $\mathrm{ABC}$ of the uterine cervix and ACC of the vulva from 1973-2014 were included in the present study. A signed Research Data Agreement was obtained to access these data. The present study was approved by the Institutional Review Board at the Johns Hopkins University School of Medicine (Baltimore, MD, USA).
Variables. The following International Classification of Diseases for Oncology (ICD-O) site codes were used: C53 for cervix uteri and C51 for vulva. The following ICD-O histology codes were used: 8200/3 for ACC and 8098/3 for ABC. Ethnicity was recorded in the SEER database as 'White,' 'Black,' 'Other: American Indian, AK Native, Asian/Pacific Islander' or 'Unknown'. Marital status was grouped as 'Married' (including common law), 'Single' (single-never married, separated, divorced or widowed), or 'Unknown'. All diagnoses were microscopically confirmed by the contributing agency. Therapy was coded as surgery, radiotherapy, surgery with radiotherapy and no/unknown. SEER staging was based on the theory of cancer growth: 'Localized' tumor was confined to the organ of origin without extension beyond the primary organ; 'Regional extension' of tumor referred to direct extension to adjacent organs or structures or spread to regional lymph nodes; the 'Distant' stage was defined when the cancer had spread to parts of the body remote from the primary tumor. The tumor size, lymph node involvement and the International Federation of Gynecology and Obstetrics (FIGO) stage information were available for the cases that were recorded from 1988-2014.

Statistical analysis. A $\chi^{2}$ test or Fisher's exact test was used to evaluate the differences between categorical data. The Wilcoxon signed-rank test was used to compare continuous data. Prognostic factors predictive of CSS and OS were analyzed using univariate and multivariate Cox proportional hazards models. CSS and OS were calculated using the Kaplan-Meier method and compared using the log-rank test. Statistical analysis was performed with SAS version 9.4 (SAS Institute, Inc., Cary, NC, USA). $\mathrm{P}<0.05$ was considered to be statistically significant. SEER*Stat (version 8.3.4; National Cancer Institute; National Institutes of Health, Bethesda, MD, USA) software was used for incidence rates calculation. All rates were age-adjusted to the 2000 US standard (18).

\section{Results}

Histologic features. Representative histologic images of uterine cervical ACCs and ABCs and vulvar ACCs are presented in Fig. 1. Whereas certain cervical ACCs (Fig. 1A) had morphologic features similar to those of the vulva (Fig. 1B), others displayed features of higher grades in appearance, characterized by larger, less uniform nuclei with evident nucleoli and readily identified mitotic figures and apoptotic bodies. The cervical ABCs contained low-grade adenoid basal epithelioma components characterized by discrete nests of tumor in which the surrounding stroma lacked a desmoplastic reaction and the cytologic features were uniform/bland and basaloid in appearance (Fig. 1C). The cervical ACCs exhibited a diffuse p16 staining pattern, consistent with high-risk HPV-associated etiology (Fig. 1D). The vulvar ACCs usually displayed classic morphologic features characterized by uniform, small cells arranged in cords and nests with a cribriform pattern and the cystic lumens commonly filled with acellular basement membrane-like material (Fig. 1B). p16, a surrogate marker for high-risk HPV infection, mostly exhibited a focal and patchy staining pattern (Fig. 1E). Nearly all of the cervical ABCs exhibited a diffuse p16 staining pattern (Fig. 1F) associated with high-risk HPV infection. 
Table I. Clinicopathological characteristics.

\begin{tabular}{|c|c|c|c|c|c|}
\hline Characteristic & Cervical ACC & Cervical ABC & P-value & Vulvar ACC & P-value ${ }^{a}$ \\
\hline Total patients (n) & 84 & 78 & & 71 & \\
\hline Median age at diagnosis, years (range) & $72(30-90)$ & $69(28-89)$ & 0.1236 & $59(31-95)$ & $<0.0001$ \\
\hline Ethnicity, n (\%) & & & 0.0002 & & 0.0002 \\
\hline White & $48(57.1)$ & $55(70.5)$ & & $60(84.5)$ & \\
\hline Black & $32(38.1)$ & $9(11.5)$ & & $6(8.5)$ & \\
\hline Others & $4(4.8)$ & $14(18.0)$ & & $5(7.0)$ & \\
\hline Marital status, n (\%) & & & 0.0094 & & $<0.0001$ \\
\hline Married & $15(17.9)$ & $29(37.2)$ & & $40(56.3)$ & \\
\hline Single $^{b}$ & $68(81.0)$ & $45(57.7)$ & & $28(39.5)$ & \\
\hline Unknown & $1(1.1)$ & $4(5.1)$ & & $3(4.2)$ & \\
\hline SEER stage, n (\%) & & & 0.0002 & & 0.2638 \\
\hline Localized & $46(54.8)$ & $68(87.2)$ & & $45(63.4)$ & \\
\hline Regional & $29(34.5)$ & $8(10.3)$ & & $19(26.7)$ & \\
\hline Distant & $3(3.6)$ & $0(0.0)$ & & $6(8.5)$ & \\
\hline Unknown & $6(7.1)$ & $2(2.5)$ & & $1(1.4)$ & \\
\hline Surgery, n (\%) & & & $<0.0001$ & & $<0.0001$ \\
\hline Yes & $55(65.4)$ & $77(98.7)$ & & $68(95.8)$ & \\
\hline No & $24(28.6)$ & $1(1.3)$ & & $3(4.2)$ & \\
\hline Unknown & $5(6.0)$ & $0(0.0)$ & & $0(0.0)$ & \\
\hline Radiation, n (\%) & & & $<0.0001$ & & 0.0381 \\
\hline Yes & $49(58.3)$ & $12(15.4)$ & & $28(39.4)$ & \\
\hline No/unknown & $35(41.7)$ & $66(84.6)$ & & $43(60.6)$ & \\
\hline Surgery and radiation, $\mathrm{n}(\%)$ & & & 0.1830 & & 0.4469 \\
\hline Yes & $22(26.2)$ & $12(15.4)$ & & $25(35.2)$ & \\
\hline No/Unknown & $62(73.8)$ & $66(84.6)$ & & $46(64.8)$ & \\
\hline Available FIGO stage, $\mathrm{n}(\%)^{\mathrm{c}}$ & $56(100)$ & $76(100)$ & $<0.0001$ & $55(100)$ & 0.3041 \\
\hline I & $33(58.9)$ & $74(97.4)$ & & $29(52.7)$ & \\
\hline II & $14(25.0)$ & $1(1.3)$ & & $8(14.5)$ & \\
\hline III & $6(10.7)$ & $1(1.3)$ & & $14(25.5)$ & \\
\hline IV & $3(5.4)$ & $0(0.0)$ & & $4(7.3)$ & \\
\hline Tumor size $^{c}$ & & & $<0.0001$ & & 0.9344 \\
\hline Available tumor size, $\mathrm{n}$ & 33 & 49 & & 48 & \\
\hline Median tumor size, cm (range) & $3.3(0.7-8.0)$ & $1.0(0.1-7.0)$ & & $3.4(0.3-9.0)$ & \\
\hline Lymph node involvement, $\mathrm{n}(\%)^{\mathrm{c}}$ & 60 & 78 & 0.0049 & 63 & 0.1143 \\
\hline No & $45(75.0)$ & $74(94.8)$ & & $56(88.9)$ & \\
\hline Yes & $1(1.7)$ & $1(1.3)$ & & $2(3.2)$ & \\
\hline Unknown & $14(23.3)$ & $3(3.9)$ & & $5(7.9)$ & \\
\hline
\end{tabular}

${ }^{a} \chi^{2}$ test or Fisher's exact test was used for the categorical variables and the Wilcoxon signed-rank test was used for continuous variables. Bonferroni's adjusted P-value was presented as cervical ABC or vulvar ACC was compared with cervical ACC. 'Including never married, separated, divorced, widowed, unmarried, or domestic partner. ${ }^{\circ}$ Data collected after 1988. ACC, adenoid cystic carcinoma; ABC, adenoid basal carcinoma; SEER, Surveillance, Epidemiology and End Results; FIGO, The International Federation of Gynecology and Obstetrics.

Clinicopathologic and therapeutic characteristics

Incidence. The age-adjusted incidence of ACC of the uterine cervix was 0.025 (white, 0.02 ; black, 0.087) per million with a white-to-black ratio of 1:4.35. Compared with cervical ACC, the age-adjusted incidence of $\mathrm{ABC}$ was slightly higher (total population, 0.064 per million; white 0.057 ; black 0.078). The incidence of vulvar ACC was 0.036 (white, 0.038; black
0.032) per million. Black people thus appeared to be more susceptible to cervical ACC and ABC.

Age distribution. From 1973-2014, the SEER database identified a total of 233 patients in the present study, including 84 cervical ACC patients, 78 with cervical ABC and 71 with vulvar ACC. The age distribution of these cases is summarized in Table I and 

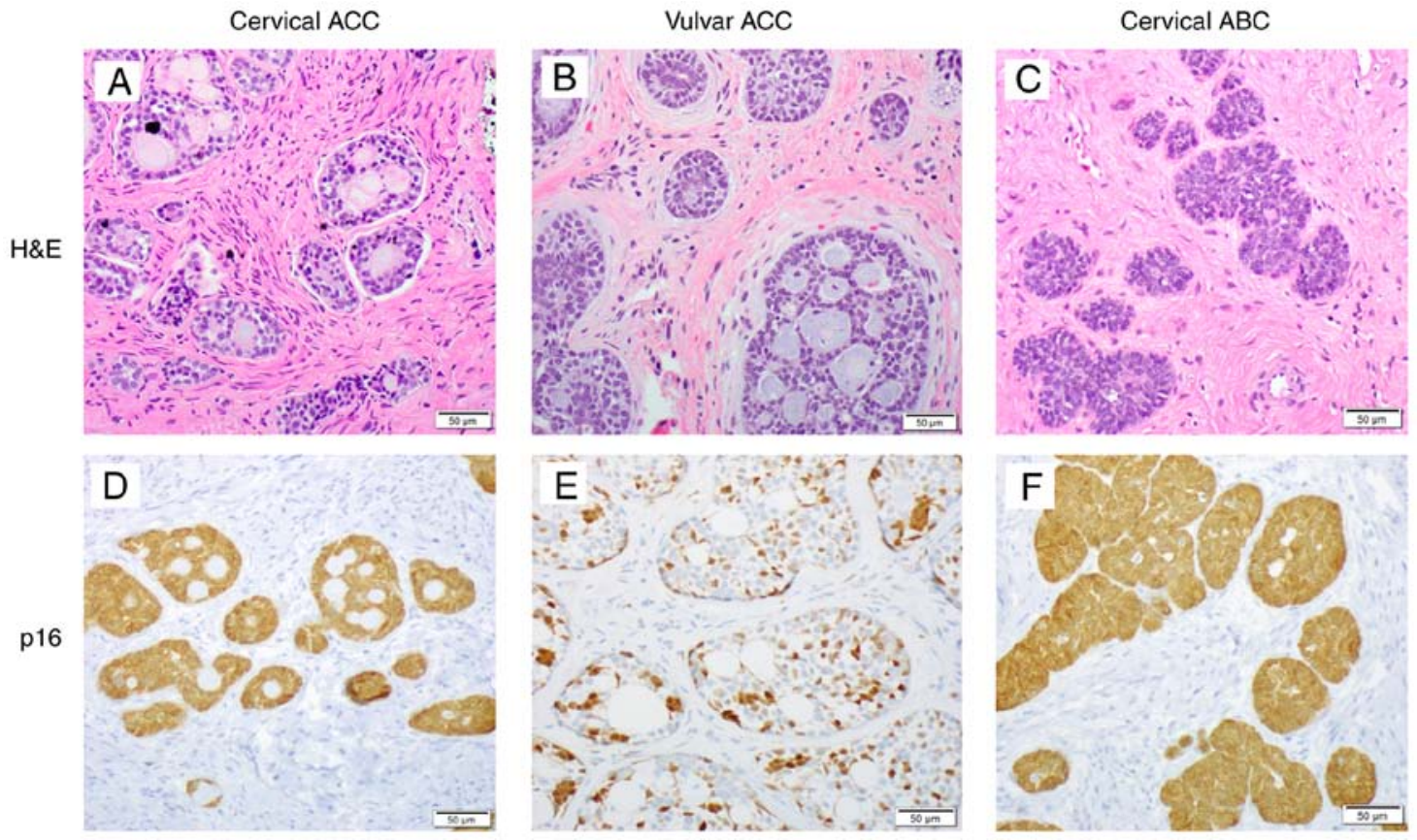

Figure 1. Representative histologic images of uterine cervical ACC, ABC and vulvar ACC. (A) Cervical ACC had morphologic features similar to those of (B) the vulva. (C) The cervical ABC was characterized by discrete nests of tumors with a uniform/bland and basaloid appearance. A diffuse p16 staining pattern was present in the cervical ACC (D) but a focal and patchy p16 staining pattern was shown in (E) the vulvar ACC. (F) Cervical ABC also displayed a diffuse p16 staining. ACC, adenoid cystic carcinoma; ABC, adenoid basal carcinoma; H\&E, hematoxylin and eosin.

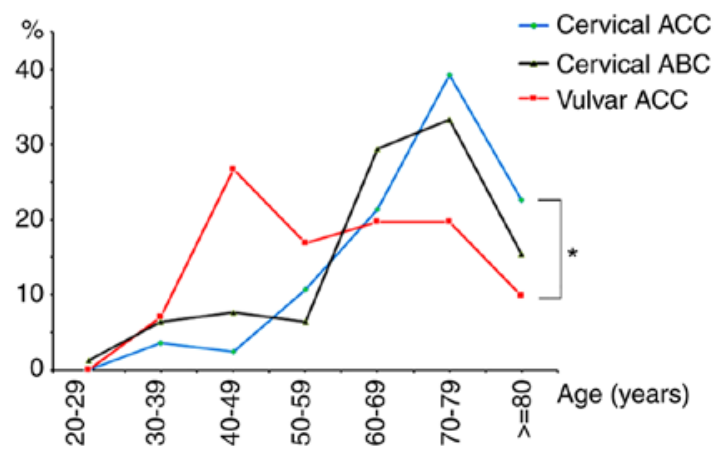

Figure 2. Age distribution of patients with cervical $\mathrm{ACC}$ and $\mathrm{ABC}$, and vulvar ACC. Patients with vulvar ACC were significantly younger than those with cervical ACC. ${ }^{*} \mathrm{P}<0.0001$. ACC, adenoid cystic carcinoma; ABC, adenoid basal carcinoma.

illustrated in Fig. 2. The ages of the patients with cervical ACC ranged from 30-90 years (median, 72 years) and $\mathrm{ABC}$ from 28-89 years (median, 69 years). With a similar age distribution pattern, the peak incidence of these two tumors was observed in the seventh and eighth decades and had no statistical difference. In contrast, the patients with vulvar ACC were significantly younger (range, 31-95 years; median, 59 years) than those with cervical ACC $(\mathrm{P}<0.0001)$. The peak incidence of vulvar ACC was observed in the fifth decade of life (Fig. 2).

Ethnicity and marital status. As presented in Table I, $32(38.1 \%)$ of 84 patients who had cervical ACC were black. In contrast, only $11.5 \%(9 / 78)$ of patients with cervical ABC and $8.5 \%(6 / 71)$ of patients with vulvar ACC were black. Overall, 15 (17.9\%) of 84 patients were married at the time of cervical ACC diagnosis. The proportion of married patients

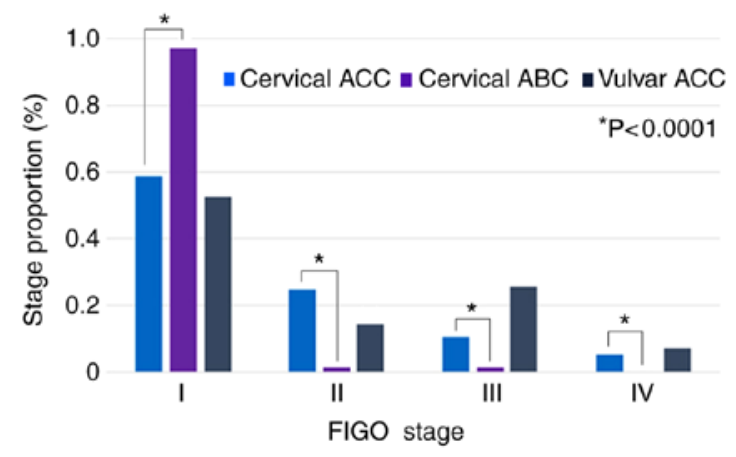

Figure 3. The FIGO stage distribution in patients with cervical ACC and ABC, and vulvar ACC. "P<0.0001. FIGO, The International Federation of Gynecology and Obstetrics; ACC, adenoid cystic carcinoma; ABC, adenoid basal carcinoma.

significantly increased in both the cervical $\mathrm{ABC}$ patients (37.2\%; $\mathrm{P}=0.0094)$ and the patients with vulvar ACC (56.3\%; $\mathrm{P}<0.0001)$ compared with those with cervical ACC (Table I).

Stage. Of 233 patients in the present study, 224 with SEER stage were available and are detailed in Table I. Of the patients with cervical ACC, 46 (54.8\%) had localized disease, 29 (34.5\%) had regional stage, and $3(3.6 \%)$ had distant metastatic disease. Whereas the patients with vulvar ACC displayed a similar stage distribution (63.4\% localized, $26.7 \%$ regional and $8.5 \%$ distant) to those with cervical ACC, the vast majority of patients with cervical $\mathrm{ABC}$ had localized disease (87.2\% localized, $10.3 \%$ regional, and $0 \%$ distant; $\mathrm{P}=0.0002$ ). A total of 187 patients (cervical ACC, 56; cervical ABC, 76; and vulvar ACC, 55) with FIGO stage were available for analysis. The distribution of FIGO stage in different types of tumor is presented in Fig. 3. 
Table II. Univariate and multivariate analysis of the 5-year hazard ratio of cause-specific survival.

\begin{tabular}{|c|c|c|c|c|c|c|}
\hline \multirow[b]{2}{*}{ Characteristic } & \multicolumn{2}{|c|}{ Cervical ACC } & \multicolumn{2}{|c|}{ Cervical ABC } & \multicolumn{2}{|c|}{ Vulvar ACC } \\
\hline & Univariate & Multivariate & Univariate & Multivariate & Univariate & Multivariate \\
\hline Age at diagnosis & $\begin{array}{c}1.07 \\
(1.02-1.13)^{\mathrm{a}}\end{array}$ & $\begin{array}{c}1.06 \\
(0.99-1.12)\end{array}$ & $\begin{array}{c}1.08 \\
(0.99-1.18)\end{array}$ & $\begin{array}{c}1.21 \\
(0.95-1.56)\end{array}$ & $\begin{array}{c}1.07 \\
(1.01-1.12)^{\mathrm{a}}\end{array}$ & $\begin{array}{c}1.10 \\
(1.02-1.19)^{\mathrm{a}}\end{array}$ \\
\hline \multicolumn{7}{|l|}{ Ethnicity } \\
\hline White & Reference & & Reference & & Reference & \\
\hline Black & $\begin{array}{c}2.35 \\
(1.03-5.36)^{\mathrm{a}}\end{array}$ & $\begin{array}{c}2.14 \\
(0.89-5.18)\end{array}$ & - & - & - & - \\
\hline Other & - & - & $\begin{array}{c}4.23 \\
(0.26-67.8)\end{array}$ & $\begin{array}{c}9.14 \\
(0.51-165.7)\end{array}$ & $\begin{array}{c}2.28 \\
(0.27-19.6)\end{array}$ & $\begin{array}{c}1.79 \\
(0.17-19.31)\end{array}$ \\
\hline \multicolumn{7}{|l|}{ Marital status } \\
\hline Married & Reference & & Reference & & Reference & \\
\hline Single $^{b}$ & $\begin{array}{c}0.89 \\
(0.30-2.62)\end{array}$ & $\begin{array}{c}1.20 \\
(0.30-4.84)\end{array}$ & - & - & $\begin{array}{c}0.25 \\
(0.03-2.04)\end{array}$ & $\begin{array}{c}0.03 \\
(<0.01-0.55)^{\mathrm{a}}\end{array}$ \\
\hline Unknown & - & - & - & - & - & - \\
\hline \multicolumn{7}{|l|}{ SEER stage } \\
\hline Localized & Reference & & Reference & & Reference & \\
\hline Regional & $\begin{array}{c}5.40 \\
(1.92-15.20)^{\mathrm{a}}\end{array}$ & $\begin{array}{c}3.80 \\
(1.23-11.77)^{\mathrm{a}}\end{array}$ & - & - & $\begin{array}{c}3.79 \\
(0.63-22.74)\end{array}$ & $\begin{array}{c}2.51 \\
(0.30-21.26)\end{array}$ \\
\hline Distant & $\begin{array}{c}3.63 \\
(0.42-31.06)\end{array}$ & $\begin{array}{c}4.31 \\
(0.44-41.90)\end{array}$ & - & - & $\begin{array}{c}10.9 \\
(1.54-77.60)^{\mathrm{a}}\end{array}$ & $\begin{array}{c}29.8 \\
(2.82-314.90)^{\mathrm{a}}\end{array}$ \\
\hline Unknown & - & - & - & - & - & - \\
\hline \multicolumn{7}{|l|}{ Surgery } \\
\hline Yes & Reference & & Reference & & Reference & \\
\hline No & $\begin{array}{c}2.38 \\
(0.97-5.88)\end{array}$ & $\begin{array}{c}1.53 \\
(0.56-4.19)\end{array}$ & - & - & - & - \\
\hline Unknown & - & - & - & - & - & - \\
\hline
\end{tabular}

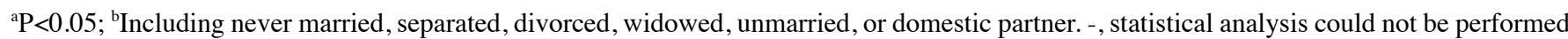
due to the small case number. ACC, adenoid cystic carcinoma; ABC, adenoid basal carcinoma; SEER, Surveillance, Epidemiology and End Results.

Overall, $33(58.9 \%)$ of 56 patients with cervical ACC had FIGO stage I disease, 14 (25.0\%) had stage II, 6 (10.7\%) had stage III and $3(5.4 \%)$ had stage IV. Similar to the stage distribution of the patients with cervical ACC $(\mathrm{P}=0.3041)$, the stage distribution of the patients with vulvar ACC was 52.7\% (29/55) stage I, $14.5 \%(8 / 55)$ stage II, $25.5 \%$ (14/55) stage III and 7.3\% (4/55) stage IV. Only $2(2.6 \%)$ of 76 cervical ABC patients were stage II and above (97.4\% stage I, 1.3\% stage II, 1.3\% stage III and $0 \%$ stage IV), which was statistically significant compared with cervical ACC $(\mathrm{P}<0.0001)$.

Tumor size and lymph node involvement. A total of 33 cervical ACC patients had known tumor sizes ranging from $0.7-8.0 \mathrm{~cm}$ (median, $3.3 \mathrm{~cm}$; Table I). The median tumor size of 48 vulvar ACCs was $3.4 \mathrm{~cm}$ (range, $0.3-9.0 \mathrm{~cm}$ ), similar to that of the cervical ACCs. Commonly discovered as an incidental finding, the known tumor size of 49 patients with cervical ABCs ranged from 0.1-7.0 cm (median, $1.0 \mathrm{~cm}$ ), significantly smaller than the cervical ACCs $(\mathrm{P}<0.0001)$. Lymph node association is a very uncommon event in these tumors, with 1 case in the patients with cervical ACC, 1 case in the patients with cervical $\mathrm{ABC}$ and 2 cases in the patients with vulvar $\mathrm{ACC}$, respectively.

Treatment. Whereas $77(98.7 \%)$ of 78 patients with cervical ABC and $68(95.8 \%)$ of 71 patients with vulvar ACC underwent surgery, only $55(65.4 \%)$ of 84 cervical ACC patients were treated with surgery $(\mathrm{P}<0.0001)$. In contrast to a low surgery rate, more cervical ACC patients received radiation therapy $(49 / 84 ; 58.3 \%)$ compared with the cervical ABC patients $(12 / 78 ; 15.4 \% ; \mathrm{P}<0.0001)$ and vulvar $\mathrm{ACC}$ patients $(28 / 71 ; 39.4 \% ; \mathrm{P}=0.0381)$. The number of patients receiving both surgery and radiation was $22(26.2 \%)$ of 84 with cervical ACC, $12(15.4 \%)$ of 78 with cervical ABC and 25 (35.2\%) of 71 with vulvar ACC, respectively.

Prognostic factors. Among all clinicopathologic variables analyzed in the cervical ACCs, the factors significantly associated with 5-year cause-specific survival (CSS) by univariate analysis were age, ethnicity and SEER stage (Table II). 
Table III. Univariate and multivariate analysis of the 5-year hazard ratio of overall survival.

\begin{tabular}{|c|c|c|c|c|c|c|}
\hline \multirow[b]{2}{*}{ Characteristic } & \multicolumn{2}{|c|}{ Cervical ACC } & \multicolumn{2}{|c|}{ Cervical ABC } & \multicolumn{2}{|c|}{ Vulvar ACC } \\
\hline & Univariate & Multivariate & Univariate & Multivariate & Univariate & Multivariate \\
\hline Age at diagnosis & $\begin{array}{c}1.06 \\
(1.02-1.11)^{\mathrm{a}}\end{array}$ & $\begin{array}{c}1.05 \\
(1.00-1.10)^{\mathrm{a}}\end{array}$ & $\begin{array}{c}1.08 \\
(0.99-1.18)\end{array}$ & $\begin{array}{c}1.08 \\
(0.97-1.20)\end{array}$ & $\begin{array}{c}1.09 \\
(1.04-1.14)^{\mathrm{a}}\end{array}$ & $\begin{array}{c}1.16 \\
(1.07-1.26)^{\mathrm{a}}\end{array}$ \\
\hline \multicolumn{7}{|l|}{ Ethnicity } \\
\hline White & Reference & & Reference & & Reference & \\
\hline Black & $\begin{array}{c}1.95 \\
(0.96-3.94)\end{array}$ & $\begin{array}{c}1.72 \\
(0.82-3.60)\end{array}$ & - & - & - & - \\
\hline Other & $\begin{array}{c}0.98 \\
(0.13-7.43)\end{array}$ & $\begin{array}{c}1.48 \\
(0.18-12.04)\end{array}$ & $\begin{array}{c}3.41 \\
(0.76-15.28)\end{array}$ & $\begin{array}{c}2.27 \\
(0.44-11.81)\end{array}$ & $\begin{array}{c}1.52 \\
(0.19-12.1)\end{array}$ & $\begin{array}{c}1.67 \\
(0.17-16.59)\end{array}$ \\
\hline \multicolumn{7}{|l|}{ Marital status } \\
\hline Married & Reference & & Reference & & Reference & \\
\hline Single ${ }^{b}$ & $\begin{array}{c}1.28 \\
(0.45-3.67)\end{array}$ & $\begin{array}{c}1.45 \\
(0.42-5.08)\end{array}$ & $\begin{array}{c}1.61 \\
(0.31-8.29)\end{array}$ & $\begin{array}{c}1.62 \\
(0.30-8.66)\end{array}$ & $\begin{array}{c}0.83 \\
(0.24-2.82)\end{array}$ & $\begin{array}{c}0.13 \\
(0.02-0.79)^{\mathrm{a}}\end{array}$ \\
\hline Unknown & - & - & - & - & - & - \\
\hline \multicolumn{7}{|l|}{ SEER stage } \\
\hline Localized & Reference & & Reference & & Reference & \\
\hline Regional & $\begin{array}{c}2.29 \\
(1.08-4.90)^{\mathrm{a}}\end{array}$ & $\begin{array}{c}1.88 \\
(0.80-4.43)\end{array}$ & $\begin{array}{c}5.82 \\
(1.30-26.03)^{\mathrm{a}}\end{array}$ & $\begin{array}{c}5.18 \\
(1.06-25.37)^{\mathrm{a}}\end{array}$ & $\begin{array}{c}2.05 \\
(0.55-7.64)\end{array}$ & $\begin{array}{c}1.07 \\
(0.21-5.49)\end{array}$ \\
\hline Distant & $\begin{array}{c}1.38 \\
(0.18-10.52)\end{array}$ & $\begin{array}{c}1.71 \\
(0.21-14.19)\end{array}$ & - & - & $\begin{array}{c}4.28 \\
(0.83-22.1)\end{array}$ & $\begin{array}{c}4.96 \\
(0.72-33.97)\end{array}$ \\
\hline Unknown & - & - & - & - & - & - \\
\hline \multicolumn{7}{|l|}{ Surgery } \\
\hline Yes & Reference & & Reference & & Reference & \\
\hline No & $\begin{array}{c}1.49 \\
(0.69-3.24)\end{array}$ & $\begin{array}{c}1.18 \\
(0.51-2.72)\end{array}$ & - & - & $\begin{array}{c}4.14 \\
(0.52-33.0)\end{array}$ & $\begin{array}{c}0.07 \\
(<0.01-3.29)\end{array}$ \\
\hline Unknown & - & - & - & - & - & - \\
\hline
\end{tabular}

${ }^{\mathrm{a}} \mathrm{P}<0.05$; ${ }^{\mathrm{b}}$ Including never married, separated, divorced, widowed, unmarried, or domestic partner. -, statistical analysis could not be performed due to the small case number. ACC, adenoid cystic carcinoma; ABC, adenoid basal carcinoma; SEER, Surveillance, Epidemiology and End Results.

Increased age, black patients and higher stages were associated with an adverse outcome. Tumor SEER stage remained an independent prognostic factor upon multivariate analysis [hazard ratio (HR) and $95 \%$ confidence interval (CI), 3.80 (1.23-11.77)]. Over a long-term follow-up, age and tumor SEER stage remained the independent prognostic factors associated with 10-year CSS of the cervical ACC patients by both univariate and multivariate analysis (data not shown). As for the 5- and 10-year OS in this group of ACC patients, increased age, as a continuous variable, was the only significant factor associated with a worse prognosis by both univariate and multivariate analysis (Table III and unpublished data).

Subsequently, the prognostic factors of the patients with vulvar ACC were analyzed. Age was significantly associated with a 5- and 10-year CSS by both univariate and multivariate analysis (Table II and unpublished data). Notably, the patients who were single appeared to have a favorable prognosis upon multivariate analysis (5-year CSS, HR 0.03, 95\% CI 0.002-0.55; 10-year CSS, HR 0.17, 95\% CI 0.03-0.86). Higher SEER stage was adversely correlated with CSS and OS survival when univariate and multivariate analyses were performed over a 10-year follow-up (multivariate CSS, HR 5.71, 95\% CI 1.11-29.37; multivariate OS, HR 4.01, 95\% CI 1.10-14.67).

Clinicopathologic factors associated with CSS and OS in the patients with cervical ABC were further analyzed. SEER stage remained as the only significant factor associated with 5-year OS by both univariate and multivariate analysis (univariate OS, HR 5.82, 95\% CI 1.30-26.03; multivariate OS, HR 5.18, 95\% CI 1.06-25.37; Table III). None of the other clinicopathologic factors reached statistical significance by all types of survival analysis.

Comparative survival outcomes. The Kaplan-Meier plots (Fig. 4) illustrate a significant difference in CSS and OS between the different types of tumor. The 5- and 10-year CSS rates in the patients with cervical ACC were 69.3 and $57.9 \%$, respectively. Compared with cervical ACC, the patients with cervical ABC had a much better prognosis, with 5- and 10-year 
A

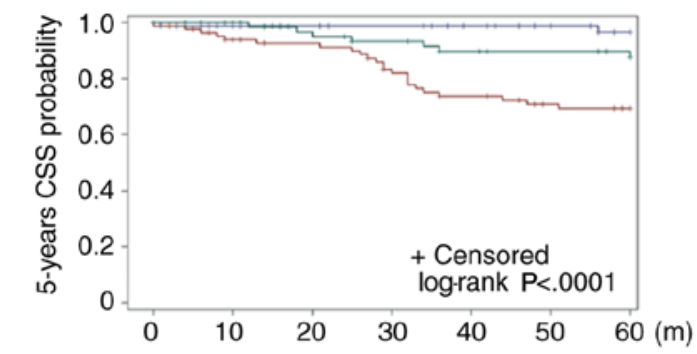

C

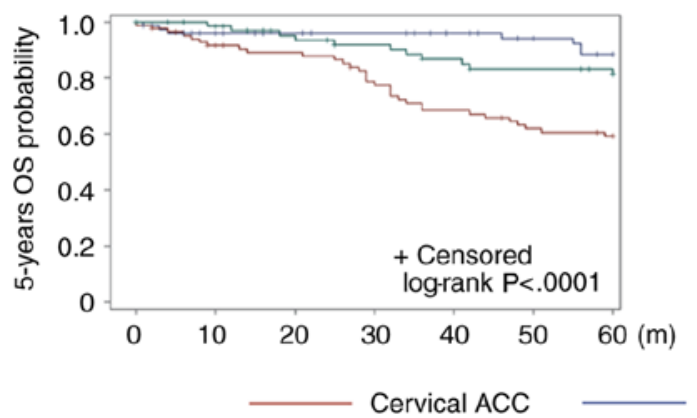

B

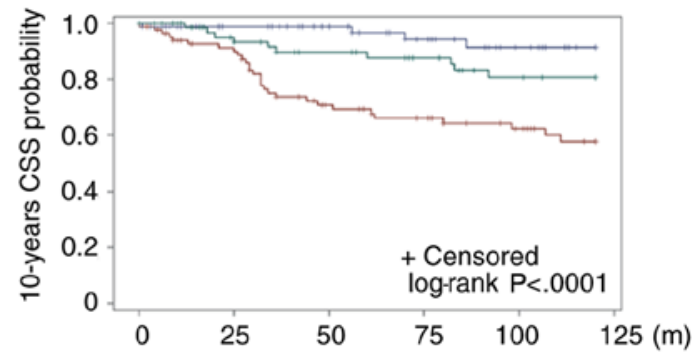

D

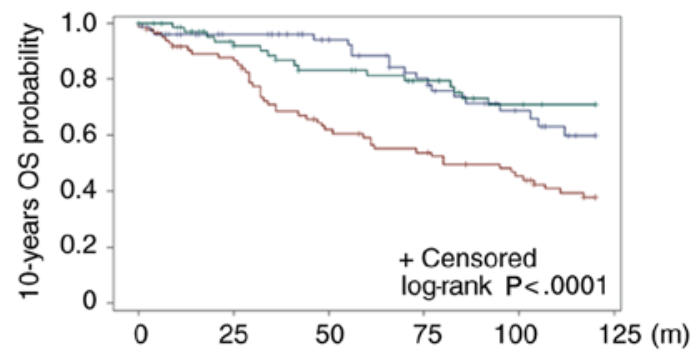

Cervical $A B C$

Vulvar ACC

Figure 4. The 5-year and 10-year CSS and OS rates of patients with cervical ACC and ABC, and vulvar ACC. (A) 5-year CSS; (B) 10-year CSS; (C) 5-year OS; (D) 10-year OS. All comparisons were statistically significant $(\mathrm{P}<0.05)$ except paired Kaplan-Meier survival analyses for cervical ABC and vulvar ACC that exhibited similar survival rates with the following P-values: 0.1 (5-year CSS); 0.08 (10-year CSS); 0.6 (5-year OS); and 0.3 (10-year OS). CSS, cause-specific survival; OS, overall survival; ACC, adenoid cystic carcinoma; ABC, adenoid basal carcinoma; m, months.

CSS rates of 96.6 and $91.4 \%(\mathrm{P}<0.0001)$, respectively. The prognosis of the vulvar ACC patients was between cervical ACC and ABC, with 5- and 10-year CSS rates of 87.7 and $80.7 \%$, respectively, which were significantly different from that of cervical ACC (5-year CSS; 10-year CSS, P=0.01), but comparable with that of $\mathrm{ABC}$ (5-year CSS, $\mathrm{P}=0.1$; 10 -year $\mathrm{CSS}, \mathrm{P}=0.08$ ). The 5- and 10-year OS rate for patients with cervical ACC was 59.2 and $37.7 \%$, respectively. The 5-year OS rates for the patients with cervical ABC $(88.3 \%)$ and vulvar ACC (81.2\%) were similar, but more favorable compared with cervical ACC (cervical ABC vs. cervical ACC, $\mathrm{P}=0.002$; vulvar ACC vs. cervical $A C C, P=0.01$ ). The 10 -year $\mathrm{OS}$ for the patients with cervical $\mathrm{ABC}$ appeared to be worse than that of vulvar ACC (59.9 vs. 71.1\%), but statistically insignificant.

\section{Discussion}

$\mathrm{ACC}$ and $\mathrm{ABC}$ in the lower female genital tract are extremely rare, illustrated by markedly low incidence rates described in the present study. Given their rarity, the majority of knowledge about these tumors is limited to case reports and small case series at a single institution. In this population-based study, clinicopathologic characteristics and prognostic factors of ACC and $\mathrm{ABC}$ in the lower female genital tract were systemically investigated. Consistent with previous findings, the results of the present study demonstrated distinct age distribution among patients with these lesions. The previously reported median ages of patients with both cervical ACC and ABC were mostly in the $60 \mathrm{~s}$ and $70 \mathrm{~s}$ (range, 50-86 years) $(12,16,19)$. Similarly, the median age of the patients with these tumors were 72 and 69 years, respectively, in the present study. It is of interest that, unlike cervical ACC and ABC, other high-risk HPV-associated tumors, such as squamous cell carcinoma or adenocarcinoma, usually occurred in the 40s and 50s $(10,20,21)$. The reason for this difference remains elusive. In a small case series study, it was demonstrated that the median age of patients with vulvar ACC was 52 years old, similar to the results of the current study (median, 59) (13). Our previous results also demonstrated that cervical carcinomas with mixed differentiation including an adenoid cystic component are high-risk HPV-associated, whereas pure ACCs of vulvar and cervical origin appear to be unrelated to high-risk HPV (12). Consistent with this finding, it has been demonstrated that $66.7 \%$ of vulvar ACCs harbored NFIB rearrangement (13). The difference in the etiology may be attributed to the distinct age distribution in these tumors.

It has been well accepted that both cervical ACC and $\mathrm{ABC}$ have a predilection to affect elderly, non-Caucasian women $(11,19)$. The present study revealed a low proportion of black patients with these tumors $(38.1 \%$ ACC and $11.5 \%$ $\mathrm{ABC}$, respectively) that appeared to contradict documented literature. Notably, the absolute number of patients of different ethnicities reflects proportionally registered patients based on the whole population selection. When the incidence rate is considered, cervical ACCs in the black population occur much more frequently compared with the Caucasian population. Similarly, the occurrence of cervical ABCs in the black population has increased 1.4 times compared with the Caucasian population. Increased occurrence of cervical ACC and ABC in the black population is postulated to be due to a relatively high HPV infection rate in this population (22). To the best of our knowledge, the ethnicity distribution of patients with vulvar ACC, an HPV-unrelated tumor, has not yet been systemically investigated. In the present study, the occurrence of vulvar ACC appeared to have no racial predilection. It is also notable 
that there was a remarkable marital status difference among the three types of tumors. The patients with cervical ACC and $\mathrm{ABC}$ tended to be unmarried compared with those with vulvar ACC. While this observation may reflect the socioeconomic and/or racial difference, the precise reason remains unknown.

Cervical ABC is usually an incidental finding in patients undergoing hysterectomy or cone biopsy for a coexistent high-grade squamous intraepithelial lesion or other reasons $(10,16,19)$. Thus, these patients are usually asymptomatic without grossly detectable masses. Not surprisingly, it was demonstrated that the median size of the cervical ABC was $1 \mathrm{~cm}$, significantly smaller than the cervical and vulvar ACCs. In the present study, the clinical presentations of cervical and vulvar ACCs included pain, abnormal bleeding, discharge and palpable masses that can be ulcerated or friable with a median size of 3.3 and $3.4 \mathrm{~cm}$, respectively. Metastasis to the lymph nodes is a very rare event for all three types of tumor, evidenced by the fact that only 4 patients had lymph node involvement among 181 patients for whom information on nodal status was available. Accordingly, the value of lymph node dissection is obscure for these tumors.

In keeping with previous studies $(19,23)$, it was demonstrated that nearly all cervical ABCs $(97.4 \%)$ were FIGO stage I. Notably, only 2 patients in the present study had a higher stage of disease; 1 patient presented with stage II and the other with stage III. In an early study, 13 of 14 women with ABC of the cervix had either stage IA or stage IB disease and all pursued a benign clinical course (19). Patients with typical histologic features of ABC typically have excellent prognosis, evidenced by 5- and 10-year CCS rates of 96.6 and $91.4 \%$, respectively, in the present study. Accordingly, the present authors suggest that the term 'adenoid basal carcinoma' does not reflect its biologic behavior and clinical outcome. It has been proposed that pure low-grade adenoid basal tumors lacking appreciable cytological atypia, mitotic activity and an infiltrative pattern in a desmoplastic stroma are designated as 'adenoid basal epitheliomas'; tumors composed of both typical low-grade adenoid basal tumor (epithelioma) and an invasive, cytologically malignant component exhibiting adenoid basal/squamous, pure squamous, and/or adenoid cystic differentiation can be diagnosed as invasive carcinomas $(16,23,24)$. Thus, the majority of adenoid basal tumors can be classified as either epithelioma or carcinoma depending on whether microscopic features of malignancy are present.

Current standard treatment protocols are not available for $\mathrm{ABC}$ and $\mathrm{ACC}$ tumors in the female genital tract. Typical cervical ABCs can be treated conservatively in that the tumor has not been associated with metastasis or tumor-related death. In the present study, 99\% of ABC patients received surgery, and of these, 12 patients also received radiation therapy. The patients who received both surgery and radiation therapy may also have a component of invasive and destructive carcinoma that was not uncommonly coexistent with typical ABC (16). Because of benign behavior, the survival of patients with cervical $\mathrm{ABC}$ is thought to not differ significantly from the general population (19). Consistently, the only significant factor associated with 5-year OS by both univariate and multivariate analysis was the SEER stage. This finding may be confounded by undefined factors as a similar result was not obtained in CSS studies.
Originally regarded as a single entity derived from progenitor reserve cells, $\mathrm{ACC}$ and $\mathrm{ABC}$ of the uterine cervix share many morphologic features, occur in older women, and are high-risk HPV-associated (11). As proposed by previous investigators, ABCs should be distinguished from ACCs in that the latter are associated with a distinctly unfavorable prognosis $(19,25-28)$. Whereas only 2 patients $(2.6 \%)$ with cervical $\mathrm{ABC}$ had a high stage of disease in the present study, $41.1 \%$ of cervical ACCs displayed aggressive behavior (FIGO stage II and higher). Notably, 3 patients had tumors that invaded the mucosa of the bladder or rectum or extended beyond the pelvis, pathologically defined as FIGO stage IV. Correlated with stratified pathologic features, the present results demonstrated that the 5- and 10-year CSS rates for patients with cervical ACC were 69.3 and $57.9 \%$, respectively. It is not surprising that a worse OS rate profile (5-year OS 59.2\%; 10-year OS 37.7\%) for these patients is the consequence of combined disease and elderly status (median age, 72 years). Furthermore, the prognosis of cervical ACCs appeared worse than that of the ACCs of the head and neck (6).

Regardless of anatomical site, the most common treatment modality of ACC is surgical resection with postoperative radiotherapy $(9,29)$. Specifically, cervical ACC cases usually follow the guidelines that are established for similarly staged patients with squamous cell carcinoma of cervix and include surgery and radiation therapy, either alone or in a combined setting (30). In the present study, $29 \%$ of the patients with cervical ACC did not receive surgery, probably due to inoperable disease in the elderly (28). Similar to ACC in other locations, cervical ACC is also thought to be a radiosensitive tumor, and radiotherapy is commonly applied to these patients (19,29,31-33). Although undefined as an adjuvant or primary treatment, chemotherapy may also benefit patients with high stage or recurrent disease $(30,34,35)$. With regard to survival, it was demonstrated that an advanced age and a high stage remained constant factors that were associated with a poor prognosis. In fact, these factors also affected the clinical outcomes of ACCs of the head and neck (6).

To the best of our knowledge there have been no previous studies that aimed to systemically characterize clinicopathologic features and survival outcomes in patients with vulvar ACC, largely due to its rarity. The present results demonstrated that, similar to cervical ACC but unlike cervical ABC, almost half of the patients had stage II and higher disease (stage I, 52.7\%; stage II, $14.5 \%$; stage III, 25.5\%; and stage IV, 7.3\%). Notably, even frequently present with high stage, the prognosis of vulvar ACC patients appeared to be similar to that of cervical $\mathrm{ABC}$ and more favorable compared with that of cervical ACC. A previous study comparatively investigated the demographics and clinical features of patients with ACC by disease site including the female genital tract (7). The study demonstrated that patients with localized ACCs of the female genital system had a 5-year disease-specific survival rate of $87.2 \%$ and a 10 -year rate of $76.8 \%$. The limitation of that study was that ACCs of the uterine cervix and vulva were analyzed as a single entity. As the oncogenic basis of cervical ACC and vulvar ACC is different (12), the interpretation of clinicopathologic features in that setting may be biased.

Similar to cervical ACC and ABC, there is no current consensus regarding the optimal treatment of vulvar ACC. 
Surgical resection of the neoplasm with clear margins is the primary treatment and local recurrence can be managed with radiotherapy (36-38). Adjuvant chemotherapy is also recommended prior to surgery or when the margins are positive/local invasion is present $(39,40)$. Unlike patients with cervical ACC, it was demonstrated that almost all patients with vulvar ACC underwent surgery and more than one-third received radiotherapy. Similar to cervical ACC, age and tumor stage were prognostic factors associated with both OS and CSS, but patients with vulvar ACC appeared to have a better prognosis compared with those with cervical ACC.

The major limitation of the present study is the lack of a centralized pathologic review. From a pathologic point of view, ABC of the cervix is an epithelial tumor composed solely of small, well-differentiated, rounded nests of basaloid cells that have scanty cytoplasm and resemble basal cell carcinomas. $\mathrm{ABC}$ is often associated with squamous intraepithelial lesions or other carcinoma subtypes (16,23,41-44). A retrospective study at our institution indicated that $\sim 30 \%$ of ABCs were associated with another type of carcinoma, including squamous cell carcinoma, ACC, and/or small cell neuroendocrine carcinoma (data not shown). Similarly, our previous study and documented literature demonstrated that cervical ACC was frequently admixed with other invasive tumors $(12,45-47)$. Accordingly, the precise diagnosis of these tumors is extremely important for the guidance of clinical management. Unfortunately, centralized pathologic review of the SEER cases to obtain a second opinion for the diagnosis is not possible. Furthermore, critical pathologic factors associated with the prognosis such as lymphovascular space invasion, resection margin status, histopathologic variability, and details of the treatment information are not available. Nevertheless, the current population-based study, rather than case reports or small case series, allows the systemic investigation of the clinicopathological features and survival outcomes of these tumors.

In conclusion, the present data demonstrated that the distinctive clinicopathologic features and survival outcomes differ significantly among cervical ABCs, cervical ACCs, and vulvar ACCs, thus providing a rationale for location/pathologic type-based management strategies. Despite dozens of case reports and small case series on clinicopathologic features in these rare tumors, the present study systemically explored the prognosis, clinical outcomes, and related pathologic factors based on a population study. The present study may lead to a prospective clinical trial to improve the management of patients with cervical ABCs, cervical ACCs and vulvar ACCs according to stratified prognostic factors.

\section{Acknowledgements}

The present study used the Surveillance, Epidemiology, and End Results (SEER) program database. The authors acknowledge the efforts of the National Cancer Institute in the creation of this database.

\section{Funding}

The present study was supported by the Career Development Award by the Cervical Cancer SPORE program (grant no. 5P50CA098252) at Johns Hopkins (DX).

\section{Availability of data and materials}

All original data and statistical code are available upon request.

\section{Authors' contributions}

DX and JL contributed to the design and implementation of the research, the analysis of the results and the writing of the manuscript. Both authors read and approved the final manuscript.

\section{Ethics approval and consent to participate}

The present study was approved by the Institutional Review Board at the Johns Hopkins University School of Medicine (Baltimore, MD, USA).

\section{Patient consent for publication}

Not applicable.

\section{Competing interests}

The authors declare that they have no competing interests.

\section{References}

1. Khan AJ, DiGiovanna MP, Ross DA, Sasaki CT, Carter D, Son YH and Haffty BG: Adenoid cystic carcinoma: A retrospective clinical review. Int J Cancer 96: 149-158, 2001.

2. Ellington CL, Goodman M, Kono SA, Grist W, Wadsworth T, Chen AY, Owonikoko T, Ramalingam S, Shin DM, Khuri FR, et al: Adenoid cystic carcinoma of the head and neck: Incidence and survival trends based on 1973-2007 surveillance, epidemiology, and end results data. Cancer 118: 4444-4451, 2012.

3. van Weert S, Bloemena E, van der Waal I, de Bree R, Rietveld DH, Kuik JD and Leemans CR: Adenoid cystic carcinoma of the head and neck: A single-center analysis of 105 consecutive cases over a 30-year period. Oral Oncol 49: 824-829, 2013.

4. Iseli TA, Karnell LH, Graham SM, Funk GF, Buatti JM, Gupta AK, Robinson RA and Hoffman HT: Role of radiotherapy in adenoid cystic carcinoma of the head and neck. J Laryngol Otol 123: 1137-1144, 2009.

5. Lloyd S, Yu JB, Wilson LD and Decker RH: Determinants and patterns of survival in adenoid cystic carcinoma of the head and neck, including an analysis of adjuvant radiation therapy. Am J Clin Oncol 34: 76-81, 2011.

6. Jang S, Patel PN, Kimple RJ and McCulloch TM: Clinical outcomes and prognostic factors of adenoid cystic carcinoma of the head and neck. Anticancer Res 37: 3045-3052, 2017.

7. Li N, Xu L, Zhao H, El-Naggar AK and Sturgis EM: A comparison of the demographics, clinical features, and survival of patients with adenoid cystic carcinoma of major and minor salivary glands versus less common sites within the surveillance, epidemiology, and end results registry. Cancer 118: 3945-3953, 2012.

8. Silverman DA, Carlson TP, Khuntia D, Bergstrom RT, Saxton J and Esclamado RM: Role for postoperative radiation therapy in adenoid cystic carcinoma of the head and neck. Laryngoscope 114: 1194-1199, 2004.

9. Coca-Pelaz A, Rodrigo JP, Bradley PJ, Vander Poorten V, Triantafyllou A, Hunt JL, Strojan P, Rinaldo A, Haigentz M Jr, Takes RP, et al: Adenoid cystic carcinoma of the head and neck-An update. Oral Oncol 51: 652-661, 2015.

10. Kurman RJ, Carcangiu ML, Herrington CS and Young RH (eds): WHO Classification of Tumors of Female Reproductive Organs. Vol 6. 4th edition. IARC Press, Lyon, 2014.

11. Grayson W, Taylor LF and Cooper K: Adenoid cystic and adenoid basal carcinoma of the uterine cervix: Comparative morphologic, mucin, and immunohistochemical profile of two rare neoplasms of putative 'reserve cell' origin. Am J Surg Pathol 23: 448-458, 1999. 
12. Xing D, Schoolmeester JK, Ren Z, Isacson C and Ronnett BM: Lower female genital tract tumors with adenoid cystic differentiation: P16 expression and high-risk HPV detection. Am J Surg Pathol 40: 529-536, 2016

13. Xing D, Bakhsh S, Melnyk N, Isacson C, Ho J, Huntsman DG, Gilks CB, Ronnett BM and Horlings HM: Frequent NFIBassociated gene rearrangement in adenoid cystic carcinoma of the vulva. Int J Gynecol Pathol 36: 289-293, 2017.

14. Jones MW, Kounelis S, Papadaki H, Bakker A, Swalsky PA and Finkelstein SD: The origin and molecular characterization of adenoid basal carcinoma of the uterine cervix. Int $\mathrm{J}$ Gynecol Pathol 16: 301-306, 1997.

15. Grayson W, Taylor LF and Cooper K: Adenoid basal carcinoma of the uterine cervix: Detection of integrated human papillomavirus in a rare tumor of putative 'reserve cell' origin. Int J Gynecol Pathol 16: 307-312, 1997.

16. Parwani AV, Smith Sehdev AE, Kurman RJ and Ronnett BM Cervical adenoid basal tumors comprised of adenoid basal epithelioma associated with various types of invasive carcinoma: Clinicopathologic features, human papillomavirus DNA detection, and P16 expression. Human pathology 36: 82-90, 2005.

17. Grayson W and Cooper K: Adenoid basal epithelioma versus adenoid basal carcinoma. Am J Surg Pathol 24: 313-314, 2000.

18. National Cancer Institute: Surveillance E, and End Results (SEER) Program (www.seer.cancer.gov) SEER*Stat Database: Incidence-SEER 9 Regs Research Data, Nov 2016 Sub (1973-2014) $<$ Katrina/Rita Population Adjustment $>$ - Linked To County Attributes-Total U.S., 1969-2015 Counties, National Cance Institute DCCPS, Surveillance Research Program, released April 2017, based on the November 2016 submission. https://seer.cancer. gov/data-software/documentation/seerstat/nov2016/. Accessed November 18, 2016

19. Ferry JA and Scully RE: 'Adenoid cystic' carcinoma and adenoid basal carcinoma of the uterine cervix. A study of 28 cases. Am J Surg Pathol 12: 134-144, 1988.

20. Small W Jr, Bacon MA, Bajaj A, Chuang LT, Fisher BJ, Harkenrider MM, Jhingran A, Kitchener HC, Mileshkin LR, Viswanathan AN, et al: Cervical cancer: A global health crisis. Cancer 123: 2404-2412, 2017.

21. Zhou J, Wu SG, Sun JY, Li FY, Lin HX, Chen QH and He ZY: Comparison of clinical outcomes of squamous cell carcinoma, adenocarcinoma, and adenosquamous carcinoma of the uterine cervix after definitive radiotherapy: A population-based analysis. J Cancer Res Clin Oncol 143: 115-122, 2017.

22. Viens LJ, Henley SJ, Watson M, Markowitz LE, Thomas CC, Thompson TD, Razzaghi H and Saraiya M: Human papillomavirus-associated cancers-United States, 2008-2012. MMWR Morb Mortal Wkly Rep 65: 661-666, 2016

23. Brainard JA and Hart WR: Adenoid basal epitheliomas of the uterine cervix: A reevaluation of distinctive cervical basaloid lesions currently classified as adenoid basal carcinoma and adenoid basal hyperplasia. Am J Surg Pathol 22: 965-975, 1998.

24. Russell MJ and Fadare O: Adenoid basal lesions of the uterine cervix: Evolving terminology and clinicopathological concepts. Diagn Pathol 1: 18, 2006.

25. Albores-Saavedra J, Manivel C, Mora A, Vuitch F, Milchgrub S and Gould E: The solid variant of adenoid cystic carcinoma of the cervix. Int J Gynecol Pathol 11: 2-10, 1992.

26. van Dinh T and Woodruff JD: Adenoid cystic and adenoid basal carcinomas of the cervix. Obstet Gynecol 65: 705-709, 1985.

27. Chen TD, Chuang HC and Lee LY: Adenoid basal carcinoma of the uterine cervix: Clinicopathologic features of 12 cases with reference to CD117 expression. Int J Gynecol Pathol 31: 25-32, 2012.

28. Dixit S, Singhal S, Vyas R, Murthy A and Baboo HA: Adenoid cystic carcinoma of the cervix. J Postgrad Med 39: 211-215, 1993

29. Bjorndal K, Krogdahl A, Therkildsen MH, Charabi B Kristensen CA, Andersen E, Schytte S, Primdahl H, Johansen J, Pedersen HB, et al: Salivary adenoid cystic carcinoma in Denmark 1990-2005: Outcome and independent prognostic factors including the benefit of radiotherapy. Results of the Danish Head and Neck Cancer Group (DAHANCA). Oral Oncol 51: 1138-1142, 2015.
30. Kaur P, Khurana A, Chauhan AK, Singh G, Kataria SP and Singh S: Adenoid cystic carcinoma of cervix: Treatment strategy. J Clin Diagn Res 7: 2596-2597, 2013.

31. Prempree T, Villasanta U and Tang CK: Management of adenoid cystic carcinoma of the uterine cervix (cylindroma): Report of six cases and reappraisal of all cases reported in the medical literature. Cancer 46: 1631-1635, 1980.

32. Koyfman SA, Abidi A, Ravichandran P, Higgins SA and Azodi M: Adenoid cystic carcinoma of the cervix. Gynecol Oncol 99: 477-480, 2005.

33. Nishida M, Nasu K, Takai N, Miyakawa I and Kashima K: Adenoid cystic carcinoma of the uterine cervix. Int J Clin Oncol 10: 198-200, 2005.

34. King LA, Talledo OE, Gallup DG, Melhus O and Otken LB: Adenoid cystic carcinoma of the cervix in women under age 40 . Gynecol Oncol 32: 26-30, 1989.

35. Phillips GL Jr and Frye LP: Adenoid cystic carcinoma of the cervix: A case report with implications for chemotherapeutic treatment. Gynecol Oncol 22: 260-262, 1985.

36. Nomura H, Nagashima M, Aoki Y and Takeshima N: Resection of the inferior pubic ramus to completely remove locally advance adenoid cystic carcinoma of Bartholin's gland. Gynecol Oncol 147: 723-724, 2017.

37. Woida FM and Ribeiro-Silva A: Adenoid cystic carcinoma of the Bartholin gland: An overview. Arch Pathol Lab Med 131: 796-798, 2007.

38. Yoon G, Kim HS, Lee YY, Kim TJ, Choi CH, Song SY, Kim BG, Bae DS and Lee JW: Analysis of clinical outcomes of patients with adenoid cystic carcinoma of Bartholin glands. Int J Clin Exp Pathol 8: 5688-5694, 2015.

39. Mousa A, Rahimi K and Warkus T: Neoadjuvant chemoradiotherapy followed by radical vulvectomy for adenoid cystic carcinoma of Bartholin's gland: A case report and review of the literature. Eur J Gynaecol Oncol 37: 113-116, 2016.

40. Yang SY, Lee JW, Kim WS, Jung KL, Lee SJ, Lee JH, Bae DS and Kim BG: Adenoid cystic carcinoma of the Bartholin's gland: Report of two cases and review of the literature. Gynecol Oncol 100: 422-425, 2006.

41. Ferry JA: Adenoid basal carcinoma of the uterine cervix: Evolution of a distinctive clinicopathologic entity. Int J Gynecol Pathol 16: 299-300, 1997.

42. Wincewicz A, Lewitowicz P, Urbaniak-Wasik S, KanczugaKoda L, Koda M, Adamczyk-Gruszka O and Sulkowski S: Adenoid basal carcinoma-like tumor combined with invasive squamous cell carcinoma foci of uterine cervix-A case report of 55-year-old woman with literature review. Rom J Morphol Embryol 55 (Suppl 3): S1225-S1230, 2014.

43. Teramoto N, Nishimura R, Saeki T, Nogawa T and Hiura M: Adenoid basal carcinoma of the uterine cervix: Report of two cases with reference to adenosquamous carcinoma. Pathology international 55: 445-452, 2005.

44. Takeshima Y, Amatya VJ, Nakayori F, Nakano T, Iwaoki Y, Daitoku K and Inai K: Co-existent carcinosarcoma and adenoid basal carcinoma of the uterine cervix and correlation with human papillomavirus infection. Int J Gynecol Pathol 21: 186-190, 2002.

45. Shi X, Wu S, Huo Z, Ling Q, Luo Y and Liang Z: Co-existing of adenoid cystic carcinoma and invasive squamous cell carcinoma of the uterine cervix: A report of 3 cases with immunohistochemical study and evaluation of human papillomavirus status. Diagn Pathol 10: 145, 2015.

46. Yannacou N, Gerolymatos A, Parissi-Mathiou P, Chranioti S and Perdikis T: Carcinosarcoma of the uterine cervix composed of an adenoid cystic carcinoma and an homologous stromal sarcoma. A case report. Eur J Gynaecol Oncol 21: 292-294, 2000.

47. Mathoulin-Portier MP, Penault-Llorca F, Labit-Bouvier C, Charafe E, Martin F, Hassoun J and Jacquemier J: Malignant mullerian mixed tumor of the uterine cervix with adenoid cystic component. Int J Gynecol Pathol 17: 91-92, 1998. 